\title{
Systems Biology paves Pathway and Potential Enzymes Predictions towards Anticancer Drug Methyl Jasmonate Biosynthesis
}

\author{
Dipankar Ghosh \\ Department of Life Sciences, Sir Alexander Fleming Building, Imperial College London SW7 2AZ, United Kingdom.
}

\begin{tabular}{|c|c|}
\hline ARTICLE INFO & ABSTRACT \\
\hline $\begin{array}{l}\text { Article history: } \\
\text { Received on: } 20 / 06 / 2017 \\
\text { Accepted on: 18/08/2017 } \\
\text { Available online: 30/09/2017 }\end{array}$ & $\begin{array}{l}\text { Methyl Jasmonate (MJ) is a potential anticancer drug along with other therapeutic importance. MJ generation is } \\
\text { predominantly dependent on plant based route which directly or indirectly interferes with ecosystem and } \\
\text { environmental concerns. However, existing microbial platforms are not yet reliable enough to fulfil industrial } \\
\text { prospects upon MJ large scale production due to lower productivity, titer and molar yield. To this end, the major }\end{array}$ \\
\hline $\begin{array}{l}\text { Key words: } \\
\text { Pathway Prediction, In silico, } \\
\text { Productivity, Systems } \\
\text { Biology, Chassis } \\
\text { Microorganisms, Methyl } \\
\text { Jasmonate. }\end{array}$ & $\begin{array}{l}\text { components following in silico systems biology tools. Main aim of this study is to establish a platform or } \\
\text { hypothesis following systems biology which has a great potential to design efficient pathway with } \\
\text { to design two most promising intermediate metabolic routes of stearic acid to oleic acid biocatalytic conversions } \\
\text { considering thermodynamic constraint towards MJ biosynthesis. Furthermore, this in silico combinatorial } \\
\text { methodology predicts most energetically favorable downstream bioconversion of oleic acid for MJ biosynthetic } \\
\text { circuit design along with novel enzymes identifications. Moreover, the future plan will be to functionalize and } \\
\text { validate the entire predicted metabolic pathway through in vivo experimentation in suitable chassis } \\
\text { microorganisms for ameliorating MJ biosynthesis. }\end{array}$ \\
\hline
\end{tabular}

\section{INTRODUCTION}

Methyl Jasmonate (MJ) is methyl ester of Jasmonic acid (JA). MJ is a stress responsive secondary regulatory metabolite and distributed ubiquitously in diverse group of plant regimes. The stresses derive either from developmental or environmental signals which up regulates MJ biosynthesis in plants. It executes several cellular regulations in different developmental cascades (growth of root, germination of seeds, ripening of fruits, plant fertility and senescence). MJ serves plant immunity in response to several biotic (insect-driven wounding, pathogens invasions) and abiotic factors (drought, lowering temperature and salinity) (Cheong and Choi, 2003).

\footnotetext{
* Corresponding Author

Tel Office: +44-2075945213.

Email:d.ghosh@imperial.ac.uk
}

MJ has first time been identified from Jasmine flower (Jasminum grandiflorum) and rosemary (Rosmarinus officinalis L.) and synthesize de novo via the Octadecanoid Pathway (OP). Furthermore, MJ structure; OP pathway resemblances to animal anti-inflammatory prostaglandins structure, and its biogenesis respectively (Wasternack and Hause, 2002). JA (MJ precursors) is also biosynthesized from plant pathogenic fungus Lasiodiplodia theobromae in 1971 (Aldridge et al., 1971). There are several microbial linage exist naturally to produce $\mathrm{MJ}$ and/or JA derivatives likely, Botryodiplodia theobromae (914.10 mg/L), Fusarium oxysporum, Aspergillus niger, Gibberella fujikuroi (2.5 $\mathrm{mg} / \mathrm{L}$ ), microalga Schizochytrium mangrovei (Dhandhukia and Thakkar, 2008; Miersch et al., 1999; Miersch et al., 1992; Miersch et al., 1993; Tsukada et al., 2010; Yue and Jiang, 2009). On the other hand, MJ and/or JA derivatives have been extracted from Jasminium grandiflorum flowers yielding only $1.25 \mathrm{mg}$ of jasmone concentrate $/ \mathrm{kg}$ flower, which contains only $0.25 \%$ of JA (Dhandhukia and Thakkar, 2007). 


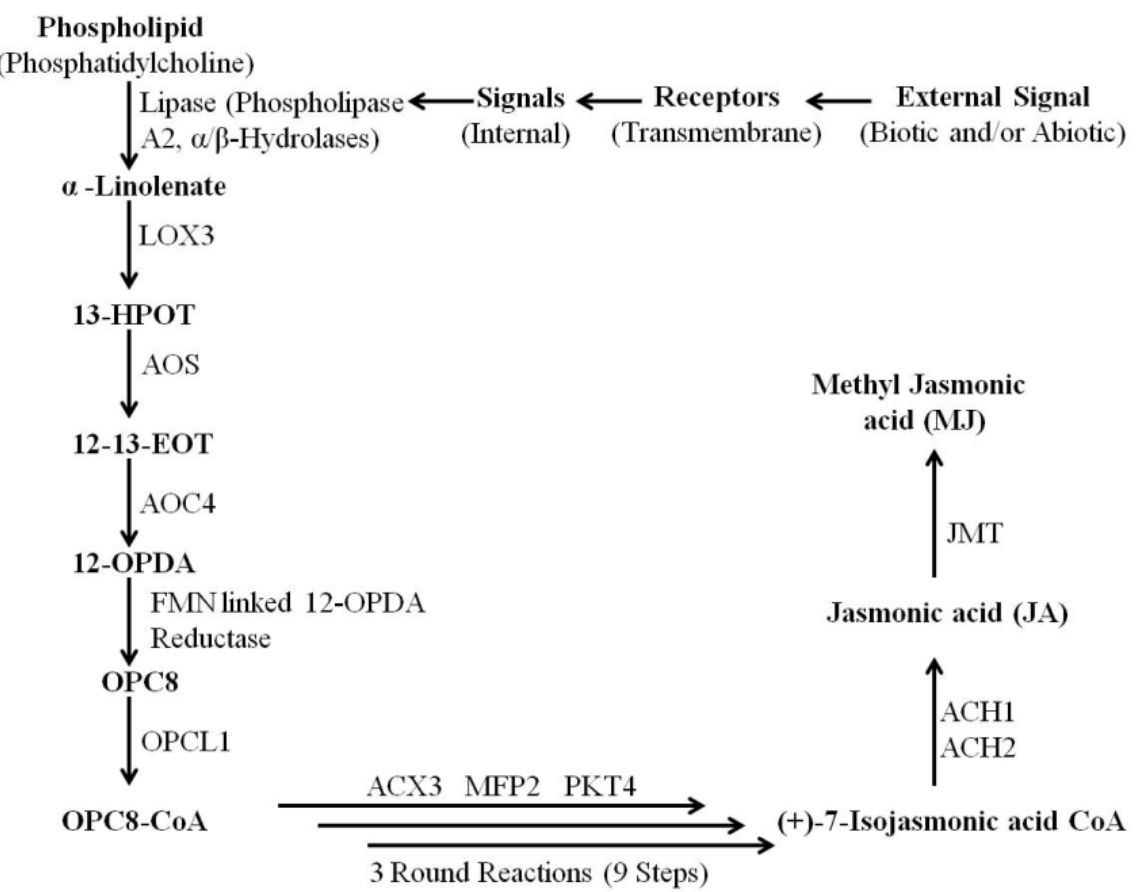

Fig 1: Schematic Diagram of biosynthetic pathway of MJ and/or JA in model plant Arabidopsis thaliana (LOX3: Lipoxygenase 3; ASO: Allene oxide synthase; AOC4: Allene oxide cyclase 4; FMN: Flavin mononucleotide; OPCL1: OPC-8:0 CoA ligase 1; 13-HPOT: (9Z,11E,15Z)-(13S)-13-Hydroperoxyoctadeca9,11,15-trienoic acid; 12-13-EOT: 12-13-(9Z,15Z)-(13S)-12,13-Epoxyoctadeca-9,11,15-trienoic acid); 12-OPDA: (15Z)-12-Oxophyto-10,15-dienoic acid; OPC8: 8-((1R,2R)-3-Oxo-2-\{(Z)-pent-2-enyl $\}$ cyclopentyl) octanoate; CoA: Coenzyme A; ACX3: Acyl-CoA oxidase 3; MFP2: Enoyl-CoA hydratase/3hydroxyacyl-CoA dehydrogenase; PKT4: Peroxisomal 3-ketoacyl-CoA thiolase 4; ACH: Acyl-CoA Thioesterase; JMT: Jasmonic Acid Carboxyl OMethyltransferase)

It has clearly been shown that MJ productivity is quiet low in its current state. However, existing microbial platforms are not yet reliable enough to fulfil industrial prospects upon MJ large scale production. Furthermore, MJ and/or JA biosynthesis has also been identified in higher plants likely Arabidopsis, tobacco, tomato and maize (Wasternack, 2007; Wasternack and Hause, 2013; Borrego and Kolomiets, 2016). However, complete biosynthetic pathways are not well characterized considering individual enzymatic reaction steps and all sets of enzymes involve. The de novo biosynthetic pathway of MJ and/or JA has been depicted in model plant Arabidopsis thaliana where this network is quiet similar with other plants and higher microbes with or without having complete enzymatic reactions and enzyme catalysts (Fig 1). Therefore, it could be a good beginning to focus on this pathway considering individual reaction and enzyme, even though give a trial may be too express and functionalize in an alternative suitable chassis or host microorganisms. But this approach is also being hindered due few bottlenecks at present scenario. Major drawbacks are likely, a. Incredibly longer reaction networks (17 reactions); b. Compartmentalization or different localizations of pathway enzymes (in peroxisome, cytosol, and chloroplast) (Reyes-Díaz et al., 2016); c. Unclear cellular regulatory systems and lack of efficient enzymes (Creelman and Mullet, 1997); d. Negative impact of MJ on photosynthesis and autotrophic carbon fixation pathways (i.e. repression of chlorophyll biosynthesis, drastic loss of chlorophyll, Rubisco degradation, increase in respiratory rate; increase in pretease and peroxidase enzyme activities) (Kode, 1992; Parthier, 1990). Moreover, MJ antagonizes the anti-senescence action of kinetin similar to abscisic acid (Ueda and Kato, 1980) in plants. MJ generally has been used as cosmetics, perfume, and flavor industries. But $\mathrm{MJ}$ recently paid much attention as therapeutic drug having anticancer activities, antidepressant, anti-aggressive activities, anti-inflammatory activities, anti-nociceptive activities, antiparasitic activities, antimicrobial, and antioxidant activities (increase in L-ascorbic acid or vitamin $\mathrm{C}$ biosynthesis) (Pirbalouti et al., 2014). Another major driving force for choosing MJ a potential drug compound that it does not cause significant acute toxicity, skin irritation, mucous membrane (eye) irritation, skin sensitization, phototoxicity, and photoallergy upon animal (including human) trials (Rotem et al., 2005; Fingrut and Flescher, 2002; Cohen and Flescher, 2009; Flescher, 2005; Umakoro and Abimbola, 2011; Scognamiglio et al., 2012). Molecular mechanism and cellular targets of MJ has been shown in great details in cancer therapy (Fig 2) (Cesai et al, 2014; Farooqi et al., 2012). However, mode of action of MJ for other therapeutic activities mentioned earlier is not pretty much clear yet. Based on this current global scenario, the major objective of this study is to identify an efficient putative metabolic pathway and its corresponding enzyme components following in-silico systems biology tools. Main aim of this study is to establish a platform or hypothesis following systems biology which has a great potential to design efficient pathway with corresponding enzymes towards improving MJ biosynthesis in near future. 

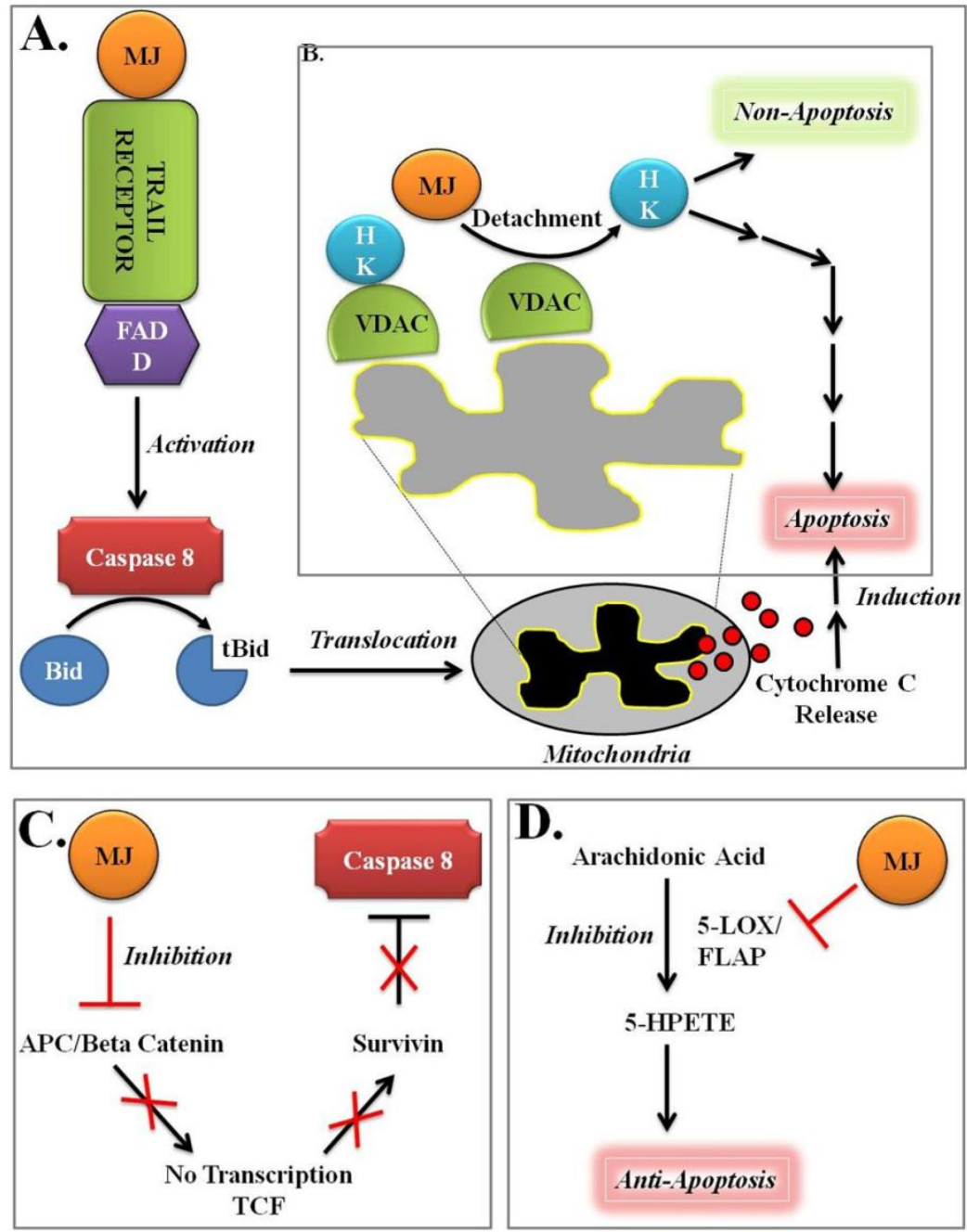

Fig 2: Mode of action of MJ as a potential anticancer drug (VDAC: Voltage-dependent anion channel ; HK: Hexokinase; APC: Antigen presenting cell; Bid: BH3 interacting-domain; tBid: truncated BH3 interacting-domain; FADD: Fas-associated death domain; TRAIL: Tumour necrosis factor-related apoptosisinducing ligand; TCF: Tumor necrotic factor; 5-LOX: 5-Lipoxygenase; ; FLAP: 5-Lipoxygenase-activating Protein; 5-HPETE: 5-Hydroperoxyeicosatetraenoic acid; ACP: Acyl carrier protein; NADH: Nicotinamide adenine dinucleotide; $\triangle \mathrm{G}$ : Gibb's free energy change; EC: Enzyme commission; CoA: Coenzyme A)

\section{MATERIALS AND METHODS}

In this current study, biosynthetic metabolic networks have been designed using Kyoto Encyclopedia of Genes and Genomes (KEGG) pathway map, eXTended metabolic space (XTMS) and eEquilibrator systems biology tools. KEGG pathway map represents a high level function of networks of molecular interactions, enzymatic reactions and relations in terms of graphical format. KEGG pathway map tool helps to draw reference pathway maps together with organism specific pathway maps that are computationally generated by matching KEGG Orthology (KO) assignments in the genome with reference pathways. KOs refers to sequence similarity groups as well as functional orthologs. Three major segments i.e. metabolic pathways, gene clusters, and phylogeny has always been considered while defining KOs (Kanehisa et al., 2017). XTMS is a rational pathway prediction tools in systems biology which is not only useful for identifying natural as well as for non-natural metabolites and novel enzymatic transformation to design biosynthetic pathways. XTMS approach determines the sets of biomolecular transformations based on specific coding system for derivation of reaction rules for enzymatic reactions, enumeration of all corresponding substrates, intermediates and products. XTMS tool provides an extended metabolic space by providing pathway ranking score, maximum allowable pathway yield and toxicity etc (Carbonell et al., 2014). Metabolic Route Explorer (MRE) is another systems biology tool used in this study to suggest foreign enzymes for the biosynthesis pathway design with competing endogenous reactions. It is an effective tool to guide the design and optimization of heterologous biosynthesis pathways providing pathway ranking scores and competing naive reactions. MRE workflow functions considering KEGG databases for biochemical transformations and Boltzmann factor for thermodynamic considerations (Kuwahara et al., 2016) within the web based 
interface. eQuilibrator is a biochemical thermodynamics calculator for individual enzymatic steps involved within the large metabolic networks. eQuilibrator is effective online systems biology search tool which connects a comprehensive and accurate database of thermodynamic properties of biochemical compounds and enzymatic reactions. It empowers easy Gibb's energies (in $\mathrm{Kcal} / \mathrm{mol}$ units) calculation of compounds and reactions considering arbitrary $\mathrm{pH}$, ionic strength and metabolite concentrations in an online interactive interface (Flamholz et al., 2012). Phylogenetic analysis has been carried out using Phylogeny.fr platform (Dereeper et al., 2008) web server (i.e. http://www.phylogeny.fr/) and MEGA5 offline software application (Tamura K et al., 2011).

\section{RESULT AND DISCUSSION}

In silico design and construction of pathways have been predicted through following simple logistic workflows (Fig 3).

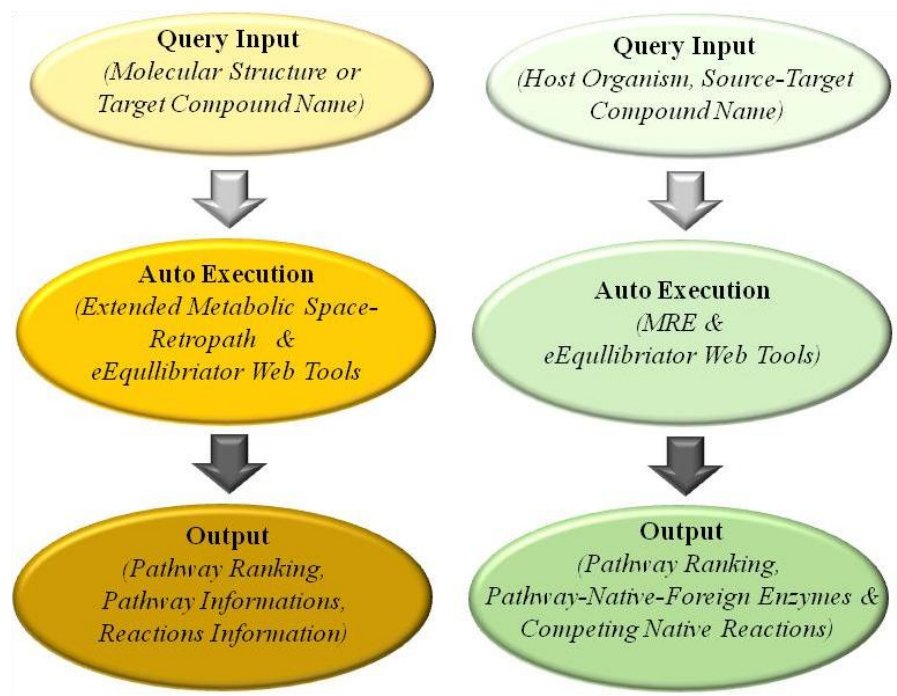

Fig 3: Systems biology (web tool based) workflow towards pathway prediction and design for MJ biosynthesis.

Core of current work flow consists of three major sections; query input, auto execution, and probable outputs. Query input considers few parameters likely host organism, source-target compound name, and molecular structure or target compound name. Secondly, auto execution segment carries out central processing following algorithms based on extended metabolic space-Retropath, MRE, and eEqullibriator web tools. Finally, output comes up with pathway ranking, pathway-reaction informations, pathway-Native-Foreign Enzymes \& competing native reactions.

Stearic acid to oleic acid conversion is the first important node of in silico design and construction of this study. In silico design not only provides shortest roots but also providing a very important list of putative enzymes for each an individual enzymatic step including thermodynamic feasibility (i.e. Gibb's free energy change). Stearic acid has been converted into Oleic acid via two nodes likely Coenzyme A (CoA) mediated route and Acyl carrier protein (ACP) mediated route (Fig 4). Stearic acid is one of the predominant chemical constituent of membrane lipids in different microbial regime and higher organisms. Under temperature stress, stearic acid has been naturally conversed into oleic acid through the enzymatic action of desaturase. This phenomenon in fatty acid composition has been extensively investigated in the mesophilic cyanobacteria likely Anabaena variabilis, Synechocystis sp. PCC 6803 and Anacystis nidulans (Kiseleva et al., 1999). Cyanobacteria carry out this accelerated unsaturation of membrane lipids to maintain the membrane fluidity which is reduced under low temperature conditions.
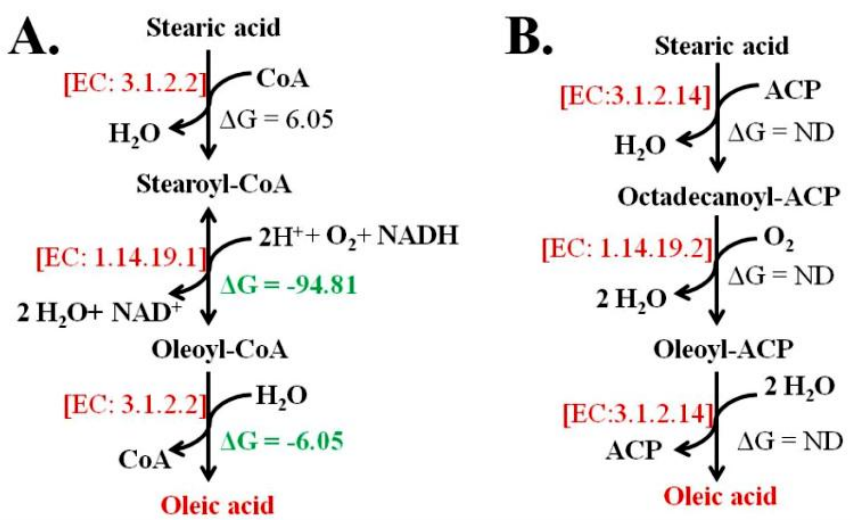

Fig 4: Predicted biosynthetic pathway design for Stearic acid to Oleic acid conversion towards MJ biosynthesis.

In this current study, CoA mediated routes seems more promising having higher negative free energy change value over ACP route. E.coli facultative anaerobic bacteria does not carry CoA metabolic route whereas Arabipsosis thaliana a plant model follows entire CoA specific route of entry of Stearic acid. While Cyanobacteria (Synechococcus sp PCC 7002 and Synechocystis sp. PCC 6803) and green algae Chlamydomonas reinhardtii bears only second anabolic step excluding other two enzymatic reactions within $\mathrm{CoA}$ route. In contrary, facultative anaerobic bacteria (E.coli), cyanobacteria (Synechococcus sp PCC 7002 and Synechocystis sp. PCC 6803) do not carry ACP mediated route. While entire ACP mediated stearic acid to oleic acid conversion route exist in Chlamydomonas reinhardtii and Arabipsosis thaliana. In ACP route, thermodynamic constraint (i.e. Gibb's free energy change) for all reactions cannot be estimated because some of the compounds don't have an explicit chemical formula. The second segment is "oleic acid to alpha-linolenate biosynthesis". In silico study has been clearly shown that this segment is slightly energetically unfavorable but several enzyme classes are involved which could be tried out under wet laboratory experimental trial towards screening potential enzymes (Fig 5). In the third phase, alpha linolenic acid has been converted into 3-oxo-2-(cis-2'pentenyl)-cyclopentane-1-octanoate (OPC8). In this segment, most of enzymatic steps are thermodynamically favorable excluding (15Z)-12-Oxophyto-10, 15-dienoic acid (12-OPDA) to OPC8 conversion (Fig 5). 


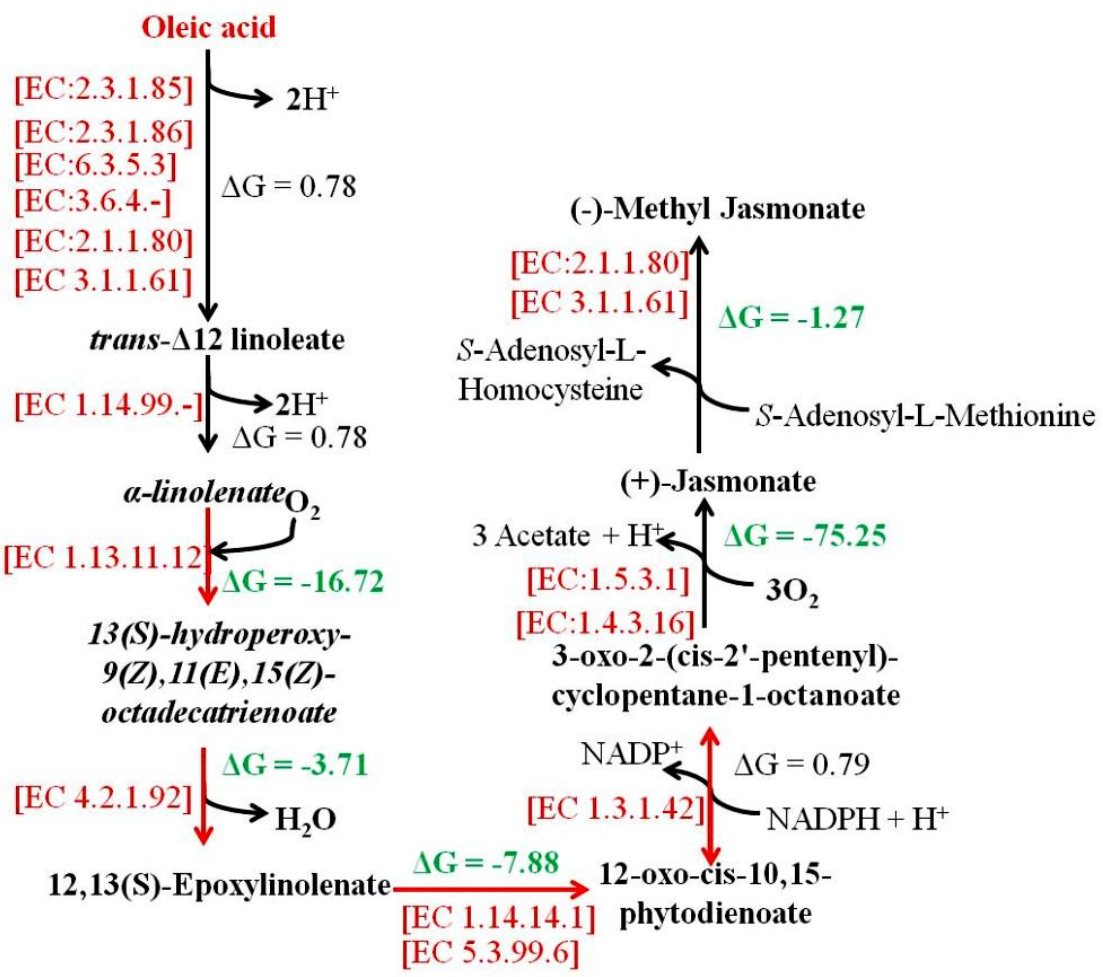

Fig 5: Predicted biosynthetic pathway design for MJ biosynthesis from stearic acid including thermodynamic constraints and putative enzyme classes.
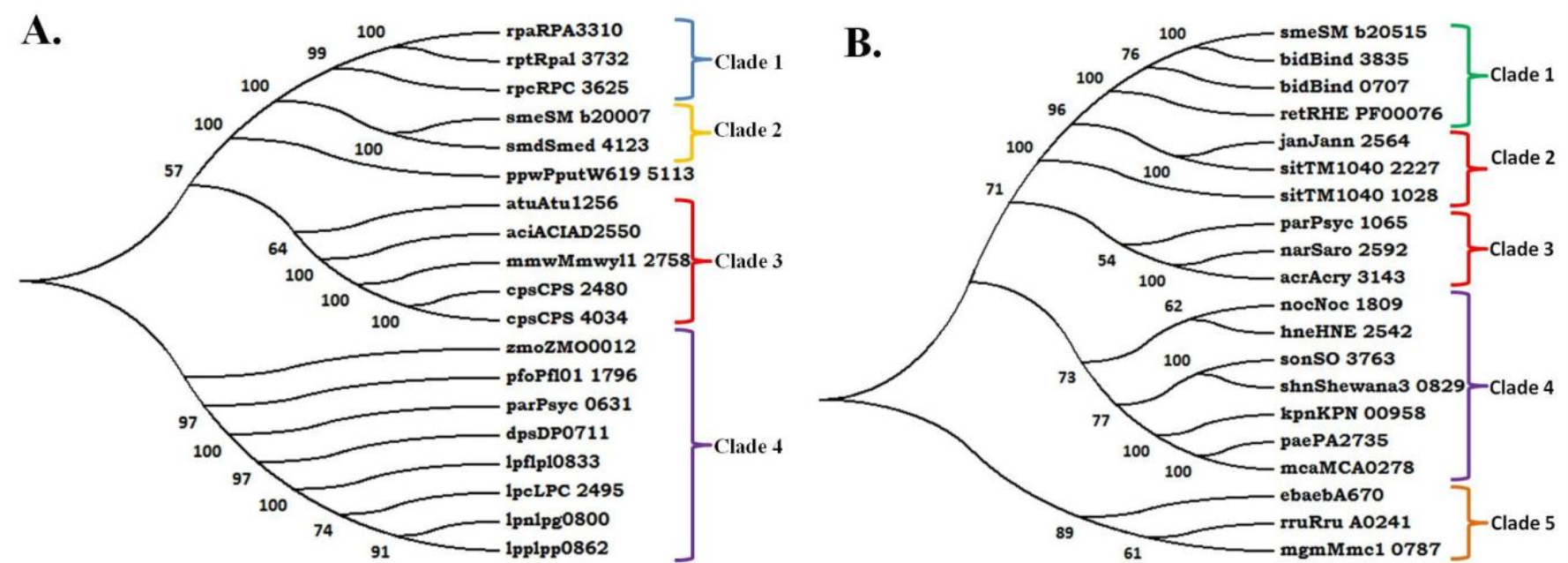

Fig 6: Phylogenetic analysis of novel enzymes involved in final two steps including (A). 3-oxo-2-(cis-2'-pentenyl)-cyclopentane-1-octanoate to JA and (B). JA to MJ conversions based on 1000 Bootstrapping and Neighbor-Joining method (aciACIAD2550: Acinetobacter sp. Sarcosine oxidase/Oxidoreductase parPsyc_0631: Psychrobacter arcticus L-Aspartate oxidase; mmwMmwyl1_2758: Marinomonas sp. Sarcosine oxidase/Oxidoreductase; pfoPfl01_1796: Pseudomonas fluorescens putative xanthine dehydrogenase; lpcLPC_2495: Legionella pneumophila L-Aspartate oxidase; lpnlpg0800: Legionella pneumophila L-Aspartate oxidase; lpplpp0862: Legionella pneumophila L-Aspartate oxidase; 1pflp10833: Legionella pneumophila L-Aspartate oxidase; dpsDP0711: Desulfotalea psychrophila L-Aspartate oxidase; rpcRPC_3625: Rhodopseudomonas palustris Catalase; smeSM_b20007: Sinorhizobium meliloti Catalase; smdSmed 4123: Sinorhizobium medicae Catalase; cpsCPS 2480: Colwellia psychrerythraea Sarcosine Oxidase; cpsCPS 4034:Colwellia psychrerythraea Sarcosine Oxidase; ppwPputW619_5113: Pseudomonas putida Catalase; zmoZMO0012: Zymomonas mobilis oxygen-independent coproporphyrinogen III oxidase; rpaRPA3310: Rhodopseudomonas palustris Catalase; rptRpal_3732: Rhodopseudomonas palustris Catalase; atuAtu1256: Agrobacterium fabrum Cytochrome P450 hydroxylase/monooxygenase; smeSM_b20515: Sinorhizobium meliloti Methyltransferase; retRHE_PF00076: Rhizobium etli Methyltransferase; sitTM1040_1028: Ruegeria sp. histidine kinase; bidBind_0707: Beijerinckia indica methyltransferase/methylesterase; bidBind_3835: Beijerinckia indica methyltransferase/methylesterase; ebaebA670: Aromatoleum aromaticum adenine-specific DNA-methyltransferase; janJann_2564: Jannaschia sp. methyltransferase/methylesterase; paePA2735: Pseudomonas aeruginosa Methyltransferases; sitTM1040_2227: Ruegeria sp. Methyltransferase; mcaMCA0278: Methylococcus capsulatus methyltransferase; nocNoc_1809: Nitrosococcus oceani methyltransferase; parPsyc_1065: Psychrobacter arcticus uroporphyrinogen-III C-methyltransferase; rruRru_A0241: Rhodospirillum rubrum Methyltransferase; narSaro_2592: Novosphingobium aromaticivorans Methyltransferase; acrAcry_3143: Acidiphilium cryptum Cyclopropane-fatty-acyl-phospholipid synthase; mgmMmc1_0787: Magnetococcus marinus Methyltransferase; kpnKPN_00958: Klebsiella pneumoniae Methylase; sonSO_3763: Shewanella oneidensis Spermidine synthase; hneHNE_2542: Hyphomonas neptunium Methyltransferase; shnShewana3_0829: Shewanella sp. spermidine synthase). 

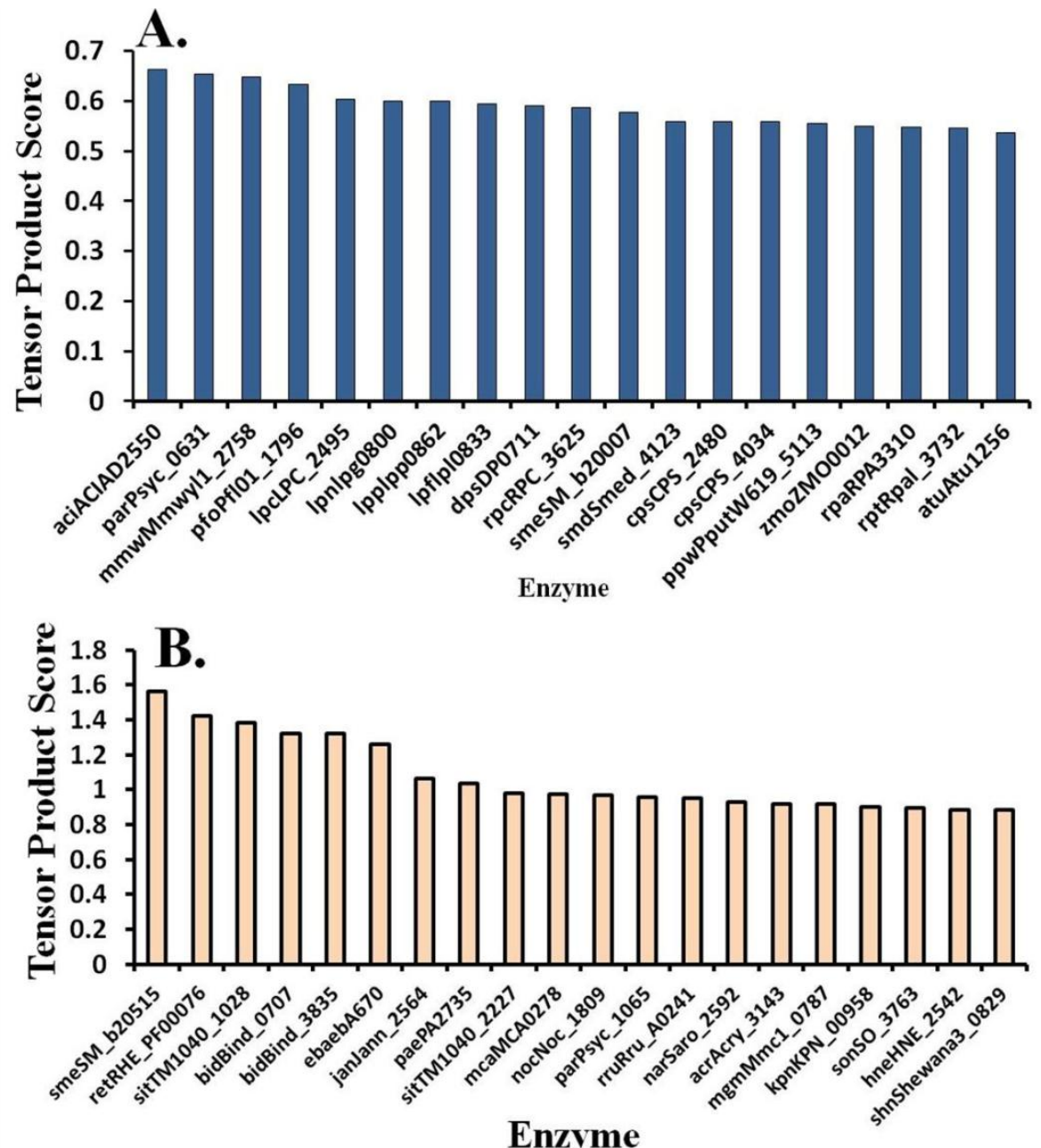

Fig 7: Biocatalytic efficacy of novel enzymes involved in final two steps including (A). 3-oxo-2-(cis-2'-pentenyl)-cyclopentane-1-octanoate to JA and (B). JA to MJ conversions based on tensor product score.

In Arabipsosis thaliana and in few higher plants, OPC8 to $\mathrm{MJ}$ and/or JA catalytic conversion requires 12 additional steps. Additionally, unavailability and/or inefficient catalytic activity of Jasmonic Acid Carboxyl O-Methyltransferase (JMT) enzyme restrict industrial production of JA via microbial route in comparison to plant based production. To this end, in silico design has shown that de novo MJ synthesis can be bypassed from OPC8 to MJ considering two most vital novel enzymatic steps (Fig. 5). These two enzymatic steps include OPC8 to (+)-JA and (+)-JA to MJ bioconversions. Furthermore, these two enzymatic steps are highly energetically favorable. Different potential enzymes involved in these reactions are classified into different groups based on phylogenetic analysis (Fig 6) and their probabilistic biocatalytic efficacy has been depicted in terms of Tensor product score (Fig 7) (Carbonell and Faulon, 2010; Faulon et al., 2008).

\section{CONCLUSION}

Synthetic Biology and Systems Biology combinatorial approach is the Holy Grail towards novel drug discovery and design of its biosynthetic pathway inside small microbial bugs (Ghosh, 2016). In over all, the current study is very strong evidence that pathway prediction and designing systems biology tools can reduce time for experimentation (i.e. trial and error conventional approach for pathway design), expenses of research, and manual effort time to time. It not only provides novel biosynthetic steps but also provides information on novel putative enzymes. In this study, predicted and designed synthetic metabolic pathways for MJ biosynthesis from stearic acid seems promising, though this entire metabolic pathway functionalization needs to be validated through in vivo experimentation in suitable chassis microorganisms (Escherichia coli, Synechococcus sp. PCC 7002, Synechococcus elongatus PCC 7942, Synechococcus sp. PCC 6803 and Chlamydomonas reinhardtii) towards industrialization via microbial biosynthetic route. Current systems biology pathway prediction and design tools in its current state could be ameliorated by connecting enzyme promiscuity and enzyme regulations phenomena in near future. However, this combinatorial methodologies could also be implemented to design novel metabolic circuits of other high value added biochemicals 
generations likely antimalarial drug Artemisinin and cholesterol lower drug 1,2,4 butanetriol. Furthermore, in silico approach enforces critical decisive role at very initial stage to define if the targeted biomolecules ought to be chosen towards further studies or not.

\section{ACKNOWLEDGEMENTS}

I would like to thank Prof. Jean-Loup Faulon for his immense support upon technical issues and web tool operations.

\section{Financial support and sponsorship: Nil.}

Conflict of Interests: There are no conflicts of interest.

\section{REFERENCES}

Aldridge DC, Galt S, Giles D, Turner WB. Metabolites of Lasiodiplodia theobromae. J Chem Soc, 1971; C: 1623-1627.

Borrego EJ, Kolomiets MV. Synthesis and Functions of Jasmonates in Maize. Plants (Basel), 2016; 5(4): 41.

Carbonell P, Faulon J-L. Molecular signatures-based prediction of enzyme promiscuity. Bioinformatics, 2010; 26: 2012-2019.

Carbonell P, Parutto P, Herisson J, Pandit SB, Faulon J-L, XTMS: pathway design in an eXTended metabolic space. Nucleic Acids Res, 2014; 42: W389-W394.

Cesari IM, Carvalho E, Rodrigues MF, Mendonça BS, Amôedo ND, Rumjanek FD. Methyl Jasmonate: Putative Mechanisms of Action on Cancer Cells Cycle, Metabolism, and Apoptosis. Int J Cell Biol, 2014; 572097: 25

Cheong J-J, Choi YD. Methyl Jasmonate as a vital substance in plants, Trends Genet, 2003; 19(7): 409-413.

Cohen S, Flescher E. Methyl Jasmonate: A Plant Stress Hormone as an Anti-Cancer Drug. Phytochemistry, 2009; 70(13-14): 1600-1609.

Creelman RA, Mullet JE. Biosynthesis and Action of Jasmonates in plants. Annu Rev Plant Physiol Plant Mol Biol, 1997; 48:355-381.

Dereeper A, Guignon V, Blanc G, Audic S, Buffet S, Chevenet F, et al. Phylogeny.fr: robust phylogenetic analysis for the non-specialist. Nucleic Acids Res, 2008; 36: W465-W469.

Dhandhukia PC, Thakkar VR. Response surface methodology to optimize the nutritional parameters for enhanced production of jasmonic acid by Lasiodiplodia theobromae. J Appl Microbiol, 2008; 105: 636-643.

Dhandhukia PC, Thakkar VR. Standardization of growth and fermentation criteria of Lasiodiplodia theobromae for production of jasmonic acid. Afr J Biotechnol, 2007; 6: 707-712.

Farooqi AA, Butt G, Razzaq Z. Algae extracts and methyl Jasmonate anti-cancer activities in prostate cancer: choreographers of 'the dance macabre'. Cancer Cell Int, 2012; 12:50.

Faulon J-L, Misra M, Martin S, Sale K, Sapra R. Genome scale enzyme-metabolite and drug target interaction predictions using the signature molecular descriptor. Bioinformatics, 2008; 24:225-233.

Fingrut O, Flescher E. Plant stress hormones suppress the proliferation and induce apoptosis in human cancer cells. Leukemia, 2002; 16: 608-616.

Flamholz A, Noor E, Bar-Even A, Milo R. eQuilibrator-the biochemical thermodynamics calculator, Nucleic Acids Res, 2012; 40: D770-D775.

Flescher E. Jasmonates a new family of anti-cancer agents. Anticancer Drugs, 2005; 16: 911-916.

Ghosh D. Synthetic Biology and Metabolic Engineering: a Workhorse for Novel Drugs Generations. Indo Am J Pharm Res, 2016; 6(11): 7012-7025.
Kanehisa M, Furumichi M, Tanabe M, Sato Y, Morishima K, KEGG: new perspectives on genomes, pathways, diseases and drugs. Nucleic Acids Res, 2017; 45: D353-D361.

Kiseleva LL, éth IH, Vigh L and Los AD. Temperature-induced specific lipid desaturation in the thermophilic cyanobacterium Synechococcus vulcanus. FEMS Microbio Lett, 1999; 175: 179-183.

Koda Y. The Role of Jasmonic Acid and Related Compounds in the Regulation of Plant Development. Int Rev Cytol, 1992; 135: 155-199.

Kuwahara H, Alazmi M, Cui X, Gao X, MRE: a web tool to suggest foreign enzymes for the biosynthesis pathway design with competing endogenous reactions in mind. Nucleic Acids Res, 2016; 44 W217-W225.

Miersch O, Bohlmann H, Wasternack C. Jasmonates and related compounds from Fusarium oxysporum. Phytochemistry, 1999; 50: 517523.

Miersch O, Bruckner B, Schmidt J, Sembdner G. Cyclopentane fatty-acids from Gibberella fujikuroi. Phytochemistry, 1992; 31: 3835 3837.

Miersch O, Günther T, Fritsche W, Sembdner G. Jasmonates from different fungal species. Nat Prod Lett, 1993; 2: 293-299.

Parthier B. Jasmonates: Hormonal regulators or stress factors in leaf senescence? J Plant Growth Regul, 1990; 9: 57-63.

Pirbalouti AG, Sajjadi SE, Parang K. A Review (Research and Patents) on Jasmonic Acid and Its Derivatives. Arch Pharm Chem Life Sci, 2014; 347: 229-239.

Reyes-Díaz M, Lobos T, Cardemil L, Nunes-Nesi A, Retamales J, Jaakola L, et al. Methyl Jasmonate: An Alternative for Improving the Quality and Health Properties of Fresh Fruits. Molecules (MDPI), 2016; 21: 567.

Rotem R, Heyfets A, Fingrut O, Blickstein D, Shaklai M, Flescher E. Jasmonates: novel anticancer agents acting directly and selectively on human cancer cell mitochondria. Cancer Res, 2005; 65: 1984-1993.

Scognamiglio J, Jones L, Letizia CS, Api AM. Fragrance Material Review on Methyl Dihydrojasmonate. Food Chem Toxicol, 2012; 50: $562-571$

Tamura K, Peterson D, Peterson N, Stecher G, Nei M, Kumar S. MEGA5: molecular evolutionary genetics analysis using maximum likelihood, evolutionary distance, and maximum parsimony methods. Mol Biol Evol, 2011; 28: 2731-2739.

Tsukada K, Takahashi K, Nabeta K. Biosynthesis of jasmonic acid in a plant pathogenic fungus, Lasiodiplodia theobromae Phytochemistry, 2010; 71: 2019-2023.

Ueda J, Kato J. Isolation and Identification of a Senescencepromoting Substance from Wormwood (Artemisia absinthium L.). Plant Physiol, 1980; 66: 246-249.

Umukoro S, Abimbola SO. Antinociceptive effects of methyl Jasmonate in experimental animals. J Nat Med, 2011; 6: 466-470.

Wasternack C, Hause B. Jasmonates and octadecanoids: signals in plant stress responses and development. Prog Nucleic Acid Res Mol Biol, 2002; 72: 165-221.

Wasternack C, Hause B. Jasmonates: Biosynthesis, perception, signal transduction and action in plant stress response, growth and development. An update to the 2007 review in annals of botany. Ann Bot, 2013; 111: 1021-1058.

Wasternack C. Jasmonates: An update on biosynthesis, signal transduction and action in plant stress response, growth and development Ann Bot, 2007; 100: 681-697.

Yue CJ, Jiang Y. Impact of methyl Jasmonate on Squalene biosynthesis in microalga Schizochytrium mangrovei. Process Biochem, 2009; 44: 923-927.

\section{How to cite this article:}

Ghosh D. Systems Biology paves Pathway and Potential Enzymes Predictions towards Anticancer Drug Methyl Jasmonate Biosynthesis. J App Pharm Sci, 2017; 7 (09): 153-159. 